\title{
Improvement of bovine ß-lactoglobulin production and secretion by Lactococcus lactis
}

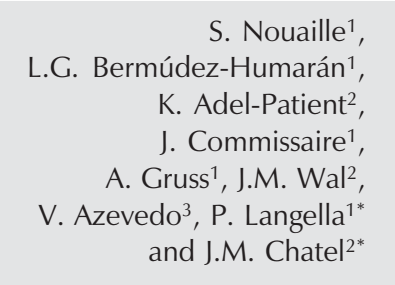

\author{
${ }^{1}$ Dairy Research and Applied Genetics Unit, INRA, Domaine de Vilvert, \\ Jouy en Josas cedex, France \\ ${ }^{2}$ Food Immunoallergy Laboratory, INRA-CEA, DRM-SPI, CEA de Saclay, \\ Gif sur Yvette, France \\ ${ }^{3}$ Instituto de Ciências Biológicas, Universidade Federal de Minas Gerais, \\ Belo Horizonte, MG, Brasil
}

\section{Correspondence \\ P. Langella \\ Ecology and Physiology of \\ Digestive Tract Unit, INRA \\ Domaine de Vilvert, 78352 \\ Jouy en Josas cedex \\ France \\ E-mail: philippe.langella@jouy.inra.fr \\ *P. Langella and J.M. Chatel \\ share the same credit as senior \\ authors of this research. \\ $\ldots \ldots \ldots \ldots \ldots \ldots$}

Received August 9, 2004

Accepted November 30, 2004

\begin{abstract}
The stabilizing effects of staphylococcal nuclease (Nuc) and of a synthetic propeptide (LEISSTCDA, hereafter called LEISS) on the production of a model food allergen, bovine ß-lactoglobulin (BLG), in Lactococcus lactis were investigated. The fusion of Nuc to BLG (NucBLG) results in higher production and secretion of the hybrid protein. When LEISS was fused to BLG, the production of the resulting protein LEISS-BLG was only slightly improved compared to the one obtained with Nuc-BLG. However, the secretion of LEISS-BLG was dramatically enhanced ( 10 - and 4-fold higher than BLG and NucBLG, respectively). Finally, the fusion of LEISS to Nuc-BLG resulting in the protein LEISS-Nuc-BLG led to the highest production of the hybrid protein, estimated at $\sim 8 \mu \mathrm{g} / \mathrm{ml}$ ( $\sim 2$-fold higher than Nuc-BLG). In conclusion, the fusions described here led to the improvement of the production and secretion of BLG. These tools will be used to modulate the immune response against BLG via delivery of recombinant lactococci at the mucosal level, in a mouse model of cow's milk allergy.
\end{abstract}

\section{Introduction}

Cow's milk allergy represents an important health problem worldwide, affecting 2 to $3 \%$ of all infants in the first two years of life (1). Bovine $\beta$-lactoglobulin (BLG) is the most abundant whey protein of cow's milk, and is regarded as a dominant allergen. BLG is a 162-amino acid globular protein, which contains two intramolecular disulfide bonds. We are interested in the production of BLG by recombinant bacteria to study the immunoproperties of this allergen and to evaluate the effect of administration of these bacteria
Key words

- Lactococcus lactis

- Protein secretion

- LEISSTCDA

- Staphylococcal nuclease

- ß-lactoglobulin

- Allergen on the modulation of the immune response in a murine model (2-4). Production of BLG by Escherichia coli results essentially in a denatured form of the protein (3). Obtaining a recombinant BLG is of great interest for the study of the relationship between the structure and the immunoreactivity of a major allergen. Moreover, expression of this allergen in non-pathogenic bacteria could be of interest for the immunomodulation of allergic reactions. BLG production in the lactic acid bacterium Lactococcus lactis (a foodgrade bacterium widely used in the food industry) results in $2 \mathrm{mg} / 1$ recombinant pro- 
tein (10- to 100-fold less than the BLG production obtained in E. coli (3). In these two bacteria, recombinant BLG is mostly produced in an intracellular and denatured form (2). Moreover, a major epitope of BLG (peptide 41-60) was successfully produced in $L$. lactis as a fusion protein with the staphylococcal nuclease (Nuc): $32.5 \mathrm{mg} / \mathrm{l} \mathrm{BLG}$ hybrid protein was then obtained (4). We now intend to improve the production and secretion of BLG by recombinant lactococci for easy recovery of high quantities of BLG from the supernatant. Moreover, the use of high-producing strains for the delivery of BLG in the gastrointestinal tract in quantities allowing the induction of a specific immune response is also a challenging possibility.

The fusion to a carrier protein (Nuc) and/ or to a synthetic propeptide, LEISSTCDA (hereafter referred to as LEISS), was repeatedly shown to stabilize and improve the production and secretion of heterologous proteins in L. lactis (5-9) when using the Nisin-Controlled Expression (NICE) $(10,11)$ system.

In the present study, we evaluated whether these tools can stabilize and improve the production and secretion of BLG by L. lactis. Our results show that the highest production was obtained with LEISS-Nuc-BLG. The fusions described in this study allow the improved production of BLG, a protein of considerable interest. This study also generates new perspectives for heterologous production of proteins poorly produced and secreted by L. lactis.

\section{Material and Methods}

\section{Bacterial strains, plasmids, and media}

The bacterial strains and plasmids used in this study are listed in Table 1. L. lactis

\begin{tabular}{|c|c|c|}
\hline Strain or plasmid & Characteristic & Reference or source \\
\hline \multicolumn{3}{|l|}{ Strains } \\
\hline E. coli TG1 & supE, hsd, $\Delta 5$, thi, $\Delta\left(\right.$ lac-proAB), $F^{\prime}$ (traD36 proAB-lacZ $\left.\Delta M 15\right)$ & Gibson, 1984 (Ref. 12) \\
\hline L. lactis MG1363 & Wild type, plasmid free & Gasson, 1983 (Ref. 13) \\
\hline L. lactis NZ9000 & MG1363 (nisRK genes into chromosome), plasmid free & Kuipers et al., 1998 (Ref. 11) \\
\hline NZ(pCYT:BLG) & NZ9000 strain producing an intracellular form of BLG protein & Chatel et al., 2001 (Ref. 2) \\
\hline NZ(pSEC:BLG) & NZ9000 strain secreting BLG protein & Chatel et al., 2001 (Ref. 2) \\
\hline NZ(pSEC:Nuc-BLG) & NZ9000 strain secreting a hybrid protein Nuc-BLG & The present study \\
\hline NZ(pSEC:LEISS-BLG) & NZ9000 strain secreting a hybrid protein LEISS-BLG & The present study \\
\hline NZ(pSEC:LEISS-Nuc-BLG) & NZ9000 strain secreting a hybrid protein LEISS-Nuc-BLG & The present study \\
\hline \multicolumn{3}{|l|}{ Plasmids } \\
\hline pBS SK II+ & Replicon ColE1, Ap ${ }^{r}$ & Stratagene \\
\hline pBS:Nsil:nuc & $\begin{array}{l}\text { Replicon ColE1, Ap }{ }^{r} \text {, PCR fragment encoding Nuc mature moiety, } \\
\text { Nsil sites at both extremities }\end{array}$ & Nouaille S (unpublished data) \\
\hline pSEC-Nuc & $\begin{array}{l}\text { Replicon pWV01, } \mathrm{Cm}^{r} \text {, gene, expressed under } \mathrm{P}_{\text {nis }} \text { encodes } \\
\text { the precursor } \mathrm{SP} \text { Usp-Nuc }\end{array}$ & Bermúdez-Humarán et al., 2003 (Ref. 7) \\
\hline pSEC:LEISS-Nuc & $\begin{array}{l}\text { Replicon pWV01, } \mathrm{Cm}^{r} \text {, gene, expressed under } \mathrm{P}_{\text {nis }} \text { encodes } \\
\text { the precursor SPUsp-LEISS-Nuc }\end{array}$ & The present study \\
\hline pSEC:Nuc-BLG & $\begin{array}{l}\text { Replicon pWV01, } \mathrm{Cm}^{r} \text {, gene, expressed under } \mathrm{P}_{\text {nis }} \text { encodes } \\
\text { the precursor } \mathrm{SP} \text { Usp-Nuc-BLG }\end{array}$ & The present study \\
\hline pSEC:LEISS-BLG & $\begin{array}{l}\text { Replicon pWV01, } \mathrm{Cm}^{r} \text {, gene, expressed under } \mathrm{P}_{\text {nis }} \text { encodes } \\
\text { the precursor SPUsp-LEISS-BLG }\end{array}$ & The present study \\
\hline pSEC:LEISS-Nuc-BLG & $\begin{array}{l}\text { Replicon pWV01, } \mathrm{Cm}^{\mathrm{r}} \text {, gene, expressed under } \mathrm{P}_{\text {nisA }} \text { encodes } \\
\text { the precursor } \mathrm{SP} \mathrm{Usp}^{-L E I S S-N u c-B L G}\end{array}$ & The present study \\
\hline
\end{tabular}

The genotypic and phenotypic characteristics are given for the strains. For the plasmids, the plasmid and cloned-cassette characteristics are given. 
was grown in M17 medium (Difco-BD, Franklin Lakes, NJ, USA) supplemented with $0.5 \%(\mathrm{w} / \mathrm{v})$ glucose at $30^{\circ} \mathrm{C}$ without shaking. E. coli was grown in $\mathrm{LB}(14)$ at $37^{\circ} \mathrm{C}$. Unless otherwise indicated, plasmid constructions were first established in E. coli TG1 and then transferred to L. lactis by electrotransformation as described (15). Plasmids were selected by the addition of antibiotics as follows: ampicillin $(100 \mu \mathrm{g} / \mathrm{ml})$ and chloramphenicol $(10 \mu \mathrm{g} / \mathrm{ml})$ for $E$. coli and chloramphenicol $(10 \mu \mathrm{g} / \mathrm{ml})$ for $L$. lactis.

\section{DNA manipulations and conditions for nisin induction}

The general procedures for DNA isolations and manipulations were essentially performed as previously described (14). Enzymes were used as recommended by the suppliers. PCR was performed using the Expand High Fidelity PCR System (Roche, Mannheim, Germany) and a Perkin Elmer Cetus apparatus (Norwalk, CO, USA). DNA sequencing was performed using a DYEnamic ET sequencing kit (Amersham, Biosciences, Piscataway, NJ, USA). For nisin promoter induction, strains were grown up to absorbance at $600 \mathrm{~nm}=0.4-0.6$ and induced with $1 \mathrm{ng}$ nisin/ml for $4 \mathrm{~h}$ (Sigma, St. Louis, MO, USA).

\section{Lactococcus lactis protein extraction and immunoblotting}

The supernatant and cell lysate fraction were prepared from induced recombinant $L$. lactis as described (2). SSD-PAGE and immunoblotting analysis with anti-Nuc or antiBLG antibodies were performed as described $(2,4)$.

\section{Fusion of Nuc carrier protein to BLG}

To test the stabilizing effect of a Nuc fusion to BLG protein, a nuc cassette harboring an $N s i$ I restriction site at both extremities was purified from Nsi I-cut pBS:Nsi I:nuc (Table 1) and cloned into Nsi I-cutpSEC:BLG backbones. The resulting plasmid pSEC:NucBLG (Figure 1) was then transferred to $L$. lactis NZ9000.

\section{Fusion of synthetic propeptide LEISS to BLG and Nuc-BLG hybrid protein}

To improve the secretion efficiency of BLG protein and Nuc-BLG hybrid protein, a DNA fragment encoding the synthetic propeptide LEISS was fused between the signal peptide and either BLG protein or Nuc-BLG hybrid protein (Figure 1). For this, we first constructed a pSEC:LEISS-Nuc plasmid, previously used by Ribeiro et al. (8). Briefly, a leiss:nuc cassette was purified from a PstIEcoRI-cut pUNuc3 plasmid (17) and cloned into a pSEC backbone purified from a PstIEcoRI-cut pSEC:Nuc (18). To generate pSEC:LEISS:BLG and pSEC:LEISS:NucBLG a blg or $n u c-b l g$ cassette was purified from an $N s i \mathrm{I} / E c o$ RI-cut pSEC:BLG or pSEC:Nuc-BLG plasmid, respectively, and cloned into an NsiI/EcoRI-cut pSEC:LEISS.

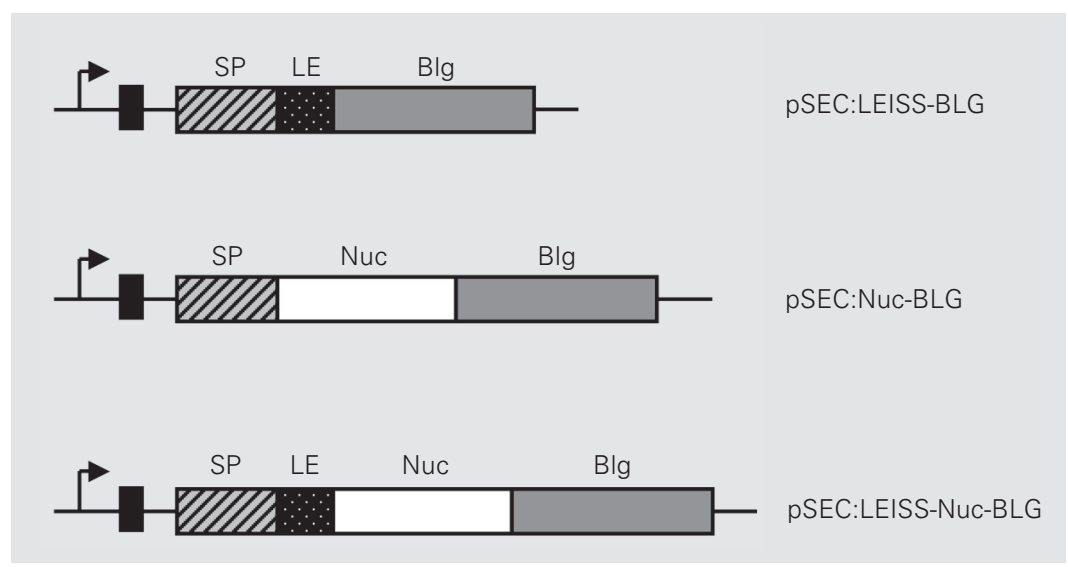

Figure 1. Expression cassettes for bovine ß-lactoglobulin (BLG) production and export in Lactococcus lactis. Schematic structures of BLG and BLG fusions expressed under control of the lactococcal nisin-inducible promoter $\left(\mathrm{P}_{\text {nis }}\right)$ and carried by the indicated plasmids are presented. The arrows indicate the $L$. lactis promoter sequence of $\mathrm{P}_{\text {nis }}$; the solid vertical bars indicate the ribosome binding sites of the usp45 gene (Ref. 16); the striped bars indicate the signal peptide of the usp45 gene (SP); the dotted bars indicate the DNA fragment encoding the LEISSTCDA (LEISS) propeptide (LE); the shaded bars indicate the region encoding $B L G(B \mid g)$; the open bars indicate the sequence encoding the staphylococcal nuclease (Nuc; not to scale). 
The resulting plasmids were then introduced into L. lactis NZ9000.

Two-site enzyme immunometric assay for BLG in native (BLGn) and denatured (BLGd) conformation

The two-site enzyme immunometric assays (EIA) for BLGn and BLGd were performed in 96-well microtiter plates (Maxisorp; Nunc, Roskilde, Denmark) essentially as described $(2,19)$. Briefly, microtiter plates were coated with a first monoclonal antibody (mAb, capture antibody) specific for BLG or BLGd. Then, $50 \mu \mathrm{l}$ of standard (BLGn or BLGd) or sample at appropriate dilution and $50 \mu \mathrm{l}$ of tracer (second $\mathrm{mAb}$ labeled with acetylcholinesterase) were added. After $18 \mathrm{~h}$ of incubation at $4^{\circ} \mathrm{C}$, the plates were extensively washed, and solidphase-bound acetylcholinesterase activity was measured by the method of Ellman (20).

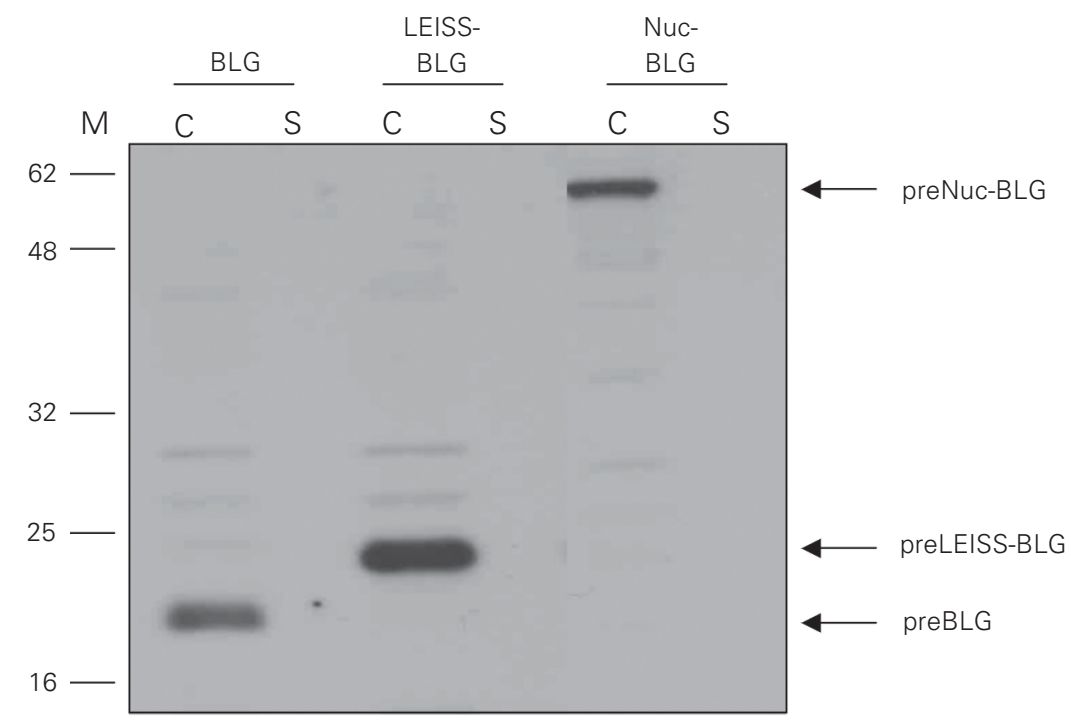

Figure 2. Production of BLG, LEISS:BLG and Nuc-BLG fusions in Lactococcus lactis was detected by Western blot with anti-BLG antibodies from induced cultures of the recombinant strains NZ9000 (pSEC:BLG), NZ9000 (pSEC:LEISS:BLG) and NZ9000 (pSEC:LEISS:NucBLG). The migration positions of the precursor forms preBLG, preLEISS:BLG and preNucBLG are indicated by arrows. BLG = bovine ß-lactoglobulin; LEISS = synthetic propeptide; $\mathrm{Nuc}=$ staphylococcal nuclease; $\mathrm{M}=$ positions and sizes of molecular mass markers $(\mathrm{kDa})$; $\mathrm{C}=$ cell lysates; $\mathrm{S}=$ supernatant fraction.

\section{Results and Discussion}

BLG production is significantly enhanced by fusion to Nuc in Lactococccus lactis

To determine the stabilizing effect of Nuc on the production of BLG in L. lactis, a recombinant $L$. lactis strain NZ9000 (pSEC: Nuc-BLG) was constructed. Western blot experiments using anti-BLG antibodies were then performed on protein extracts of cell and supernatant fractions of induced cultures of NZ9000 (pSEC:BLG) and NZ9000 (pSEC:Nuc-BLG) L. lactis strains to compare and analyze the production and the distribution of BLG and Nuc-BLG (Figure 2). As previously observed, these experiments revealed in the cell fraction of NZ9000 (pSEC:BLG) a single band corresponding to the precursor form (preBLG, $\sim 21 \mathrm{kDa}$ ) whereas no band was detected in the corresponding supernatant fraction, suggesting a weak processing and secretion of the precursor form (2) (Figure 2). This phenomenon has been previously observed for BLG. Western blot analysis of the cell fraction of the NZ9000 (pSEC:Nuc-BLG) strain revealed a protein which migrated at the expected size of the Nuc-BLG hybrid protein $(\sim 55 \mathrm{kDa})$; however, as observed for NZ9000 (pSEC: BLG), no proteins were detected in the supernatant (Figure 2). The same profile was obtained with anti-Nuc antibodies (data not shown). Thus, in order to demonstrate that $L$. lactis indeed secreted Nuc-BLG fusion, supernatant and cell lysate fractions of NZ9000 (pSEC:Nuc-BLG) were analyzed by a more accurate and sensitive test, the two-site EIA, that allows the determination of the quantity of BLG (2) (Figure 3). EIA specific for BLGn and BLGd was performed on each sample and the results are reported as ng of BLGn + BLGd. The data show that NucBLG is indeed secreted into the culture medium but at very low concentration ( $\sim 35 \mathrm{ng} /$ $\mathrm{ml}$; Figure 3B), corresponding to $\sim 2.5 \%$ secretion efficiency (the proportion of total 
protein present in mature secreted form). Total production of Nuc-BLG reached $\sim 3.5$ $\mu \mathrm{g} / \mathrm{ml}$ (Figure $3 \mathrm{~A}$ ). When compared to NZ9000 (pSEC:BLG), these results show that fusion of Nuc to the N-terminal part of the mature BLG moiety can improve the secretion of the recombinant protein (from 25 to $\sim 50 \mathrm{ng} / \mathrm{ml}$; Figure 3B). Moreover, total BLG production in L. lactis was significantly enhanced from 1500 to $\sim 3500 \mathrm{ng} / \mathrm{ml}$ (Figure $3 \mathrm{~A}$ ), whereas the secretion was only slightly improved, $\sim 50 v s \sim 25 \mathrm{ng} / \mathrm{ml}$ (Figure 3B). The BLGn/BLGd ratio was $\sim 10 \%$ in culture medium but less than $1 \%$ in terms of total production. These values are the same as those previously reported by our group (3), showing that the presence of a highly stable and secreted protein, Nuc, does not improve the folding of BLG.

\section{LEISS dramatically improves secretion efficiency and yield of BLG in Lactococcus lactis}

In previous studies, we demonstrated that the synthetic propeptide LEISS acts as a heterologous protein secretion-enhancer in L. lactis (5-8). Here, in an attempt to improve the secretion of BLG in L. lactis, we fused the propeptide LEISS between the signal peptide of the usp 45 gene (SP) and the mature moiety of the BLG protein (LEISSBLG). This fusion is encoded by an expression cassette carried by the plasmid pSEC: LEISS-BLG (Figure 1). Once this plasmid was established into L. lactis (NZ9000 (pSEC:LEISS-BLG)), protein secretion was evaluated and compared with the NZ9000 (pSEC:BLG) and NZ9000 (pSEC:Nuc-BLG) strains by immunoblotting (Figure 2) and two-site EIA (Figure 3). As observed in Figure 2, Western blot analysis with anti-BLG $\mathrm{mAb}$ revealed a single band in the cell fraction, which corresponded to the precursor form preLEISS-BLG ( $\sim 25 \mathrm{kDa})$. No BLG signal was detected in the NZ9000 (pSEC: BLG) or NZ9000 (pSEC:Nuc-BLG) strain supernatant by Western blot analysis. However, two-site EIA experiments showed a dramatic improvement of BLG secretion, with BLG concentrations in the supernatant reaching values close to $\sim 250 \mathrm{ng} / \mathrm{ml}$ (Figure $3 \mathrm{~B}$ ), which correspond to $\sim 6.5 \%$ secretion efficiency. Total protein production was calculated to be $\sim 4 \mu \mathrm{g} / \mathrm{ml}$ (Figure 3A). As was the case for Nuc-BLG, no improvement of the $\mathrm{BLGn} / \mathrm{BLGd}$ ratio was observed. BLG is almost exclusively found in a denatured conformation.

Finally, we analyzed the effect of the fusion of LEISS between the SP and mature moiety of the Nuc-BLG hybrid protein (LEISS:Nuc-BLG), encoded by the plasmid pSEC:LEISS:Nuc-BLG (Figure 1). In this case, two-site EIA (Figure 3) and Western blot (data not shown) experiments showed that secretion efficiency decreased: only $\sim 1 \%$ of the hybrid protein was detected in the supernatant fraction $v s \sim 1.5 \%$ observed for the NZ9000 (pSEC:Nuc-BLG) strain (Figure $3 \mathrm{~B})$. Interestingly, the highest yield of BLG protein $(\sim 8 \mathrm{ng} / \mathrm{ml}, 2$-fold higher than for LEISS-BLG alone) was obtained with this new fusion (LEISS-Nuc-BLG; Figure $3 \mathrm{~A})$.

We conclude that the use of L. lactis to produce and deliver BLG (a food allergen) to the gastrointestinal tract is a good poten-

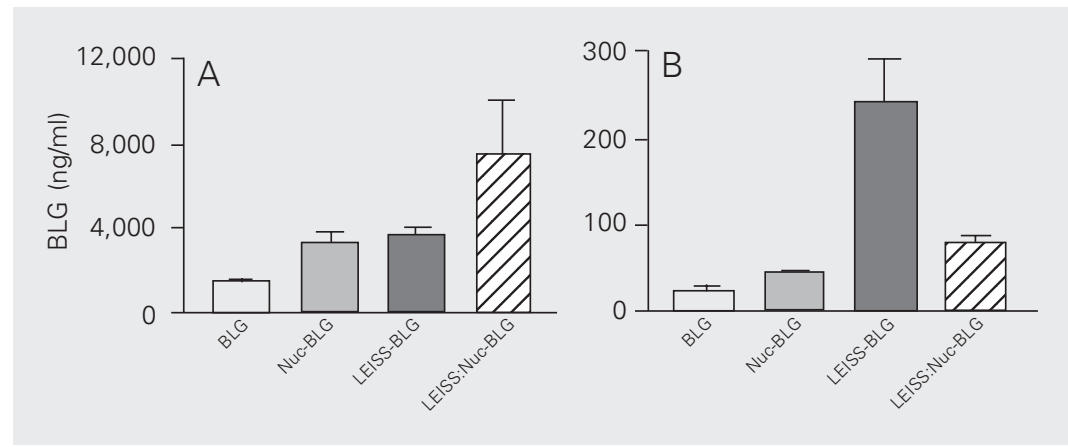

Figure 3. Production of BLG, LEISS-BLG, Nuc-BLG, and LEISS-Nuc-BLG in Lactococcus lactis was detected using two-site EIA specific for BLGn or BLGd in supernatant and cell lysate fractions from induced cultures. Data are reported as ng of BLG (BLGn + BLGd) per $\mathrm{ml}$ of induced culture. A, Total production: sum of BLG (BLGn + BLGd) detected in supernatant and cell lysate fractions. $B$, Sum of BLG (BLGn + BLGd) detected in supernatant. $B L G=$ bovine $\beta$-lactoglobulin; Nuc = staphylococcal nuclease; $L E I S S=$ synthetic propeptide. 
tial strategy to modulate the immune response against cow's milk allergy. We previously reported BLG production and secretion in L. lactis but the yields of recombinant BLG recovered in supernatant samples were very low (2). However, we have previously shown that low production and secretion efficiency of the oncoprotein E7 from human papillomavirus $(6,7)$ and the Brucella abortus antigen L7/L12 (8) in L. lactis could be enhanced by the fusion of Nuc protein and/or LEISS propeptide. Here, we found that fusion of Nuc to BLG acts in a way similar to that observed with these other two proteins and also with the VP2 protein from infectious bursal disease virus (9). Moreover, the addition of two negative charges at positions +2 and +8 of BLG by fusion of LEISS propeptide indeed improved the secretion efficiency (1.5 vs 6.5\%). In conclusion, we observed that the fusion of Nuc and LEISS significantly improves both BLG pro- duction and secretion, but not the BLGn/ BLGd ratio, reaching the highest yield with the LEISS-Nuc-BLG form (Figure 3A) and the highest secretion with the LEISS-BLG form (Figure 3A). These new strains have been used to modulate the immune response against BLG in a murine model via delivery at the mucosal level (Adel-Patient K, unpublished results). These results confirm the potential use of recombinant lactococci to treat BLG allergy. Finally, L. lactis expressing BLG could be ultimately used to easily recover large quantities of BLG from culture supernatants.

\section{Acknowledgments}

We thank Clémentine Denis for efficient technical assistance and the members of the Research and Applied Genetics Unit, INRA, Domaine de Vilvert, Jouy en Josas, France, for helpful suggestions during the study.

\section{References}

1. Host A \& Halken S (1998). Epidemiology and prevention of cow's milk allergy. Allergy, 53: 111-113.

2. Chatel JM, Langella P, Adel-Patient K, Commissaire J, Wal JM \& Corthier G (2001). Induction of mucosal immune response after intranasal or oral inoculation of mice with Lactococcus lactis producing bovine beta-lactoglobulin. Clinical and Diagnostic Laboratory Immunology, 8: 545-551.

3. Chatel JM, Adel-Patient K, Creminon C \& Wal JM (1999). Expression of a lipocalin in prokaryote and eukaryote cells: quantification and structural characterization of recombinant bovine beta-lactoglobulin. Protein Expression and Purification, 16: 70-75.

4. Chatel JM, Nouaille S, Adel-Patient K, Le Loir Y, Boe H, Gruss A, Wal JM \& Langella P (2003). Characterization of a Lactococcus lactis strain that secretes a major epitope of bovine beta-lactoglobulin and evaluation of its immunogenicity in mice. Applied and Environmental Microbiology, 69: 6620-6627.

5. Le Loir Y, Gruss A, Ehrlich SD \& Langella P (1998). A nine-residue synthetic propeptide enhances secretion efficiency of heterologous proteins in Lactococcus lactis. Journal of Bacteriology, 180: 18951903.

6. Bermúdez-Humarán LG, Langella P, Miyoshi A, Gruss A, TamezGuerra R, Montes de Oca-Luna R \& Le Loir Y (2002). Production of human papillomavirus type 16 E7 protein in Lactococcus lactis. Applied and Environmental Microbiology, 68: 917-922.

7. Bermúdez-Humarán LG, Cortes-Perez NG, Le Loir Y, Gruss A, Rodriguez-Padilla C, Saucedo-Cardenas O, Langella P \& Montes de Oca-
Luna R (2003). Fusion to a carrier protein and a synthetic propeptide enhances E7 HPV-16 production and secretion in Lactococcus lactis. Biotechnology Progress, 19: 1101-1104.

8. Ribeiro LA, Azevedo V, Le Loir Y, Oliveira SC, Dieye Y, Piard JC, Gruss A \& Langella P (2002). Efficient targeting of the Brucella abortus immunodominant antigen L7/L12 by Lactococcus lactis: a first step towards new live vaccines against brucellosis. Applied and Environmental Microbiology, 68: 910-916.

9. Dieye Y, Hoekman AJ, Clier F, Juillard V, Boot HJ \& Piard JC (2003). Ability of Lactococcus lactis to export viral capsid antigens: a crucial step for development of live vaccines. Applied and Environmental Microbiology, 69: 7281-7288.

10. de Ruyter PG, Kuipers OP \& de Vos WM (1996). Controlled gene expression systems for Lactococcus lactis with the food-grade inducer nisin. Applied and Environmental Microbiology, 62: 36623667.

11. Kuipers OP, de Ruyter PG, Kleerebezem M \& de Vos WM (1998). Quorum sensing-controlled gene expression in lactic acid bacteria. Journal of Biotechnology, 64: 15-21.

12. Gibson TJ (1984). Studies of the Epstein-Barr virus genome. PhD thesis, University of Cambridge, Cambridge, England.

13. Gasson MJ (1983). Plasmid complements of Streptococcus lactis NCDO 712 and other lactic acid streptococci after protoplast-induced curing. Journal of Bacteriology, 154: 1-9.

14. Sambrook J, Fritsch EF \& Maniatis T (1989). Molecular Cloning: A Laboratory Manual. 2nd edn. Cold Spring Harbor Laboratory, Cold 
Spring Harbor, NY, USA.

15. Langella P, Le Loir Y, Ehrlich SD \& Gruss A (1993). Efficient plasmid mobilization by pIP501 in Lactococcus lactis subsp. lactis. Journal of Biotechnology, 175: 5806-5813.

16. van Asseldonk M, Rutten G, Oteman M, Siezen RJ, de Vos WM \& Simons G (1990). Cloning, expression in Escherichia coli and characterization of usp45, a gene encoding a highly secreted protein from Lactococcus lactis MG1363. Gene, 95: 155-160.

17. Le Loir Y, Nouaille S, Commissaire J, Bretigny L, Gruss A \& Langella $P$ (2001). Signal peptide and propeptide optimization for heterologous protein secretion in Lactococcus lactis. Applied and Environmental Microbiology, 67: 4119-4127.
18. Bermúdez-Humarán LG, Langella $P$, Commissaire J, Gilbert $S$, Le Loir Y, L'Haridon R \& Corthier G (2003). Controlled intra- or extracellular production of staphylococcal nuclease and ovine omega interferon in Lactococcus lactis. FEMS Microbiology Letters, 29: 307313.

19. Negroni L, Bernard $H$, Clement G, Chatel JM, Brune P, Frobert $Y$, Wal JM \& Grassi J (1998) Two-site enzyme immunometric assays for determination of native and denatured beta-lactoglobulin. Journal of Immunological Methods, 220: 25-37.

20. Ellman GL, Courtney KD, Andres V \& Featherstone RM (1961). A new and rapid colorimetric determination of acetylcholinesterase activity. Biochemical Pharmacology, 7: 88-95. 